\title{
Screening for Diabetic Retinopathy with Extended Intervals, Safe and Without Compromising Adherence: A Retrospective Cohort Study
}

\author{
Ali Sharif (D) · Johan Jendle · Karl-Johan Hellgren
}

Received: September 14, 2020 / Accepted: October 21, 2020 / Published online: November 9, 2020

(c) The Author(s) 2020

\begin{abstract}
Introduction: Screening for diabetic retinopathy (DR) prevents blindness through the early detection of sight-threatening retinal microvascular lesions that respond to timely local treatment. However, the provision of easy and regular access to DR screening programs is currently being challenged by the increasing prevalence of diabetes. One proposed solution is to extend the screening interval for patients at low risk for progression of retinopathy. To date, most providers of screening programs have hesitated to implement a strategy of extended intervals due to the lack of data on whether adherence and safety are compromised when retinal examinations occur less frequently. In the study reported here, we investigated adherence to the screening program and progression of retinopathy in patients with type 2 diabetes participating in a DR screening program with extended intervals.
\end{abstract}

A. Sharif $(\bowtie) \cdot$ K.-J. Hellgren

Department of Ophthalmology, Karlstad Hospital, Region Värmland, Karlstad, Sweden

e-mail: ali.sharif@regionvarmland.se

A. Sharif · J. Jendle $\cdot$ K.-J. Hellgren

Diabetes Endocrinology and Metabolism Research

Center, Örebro University, Örebro, Sweden

A. Sharif · J. Jendle $\cdot$ K.-J. Hellgren Institute of Medical Sciences, Örebro University, Örebro, Sweden
Methods: This was a retrospective study that included 1000 patients with type 2 diabetes mellitus who attended a screening program for DR. The patients were consecutively placed into a low-risk patient cohort with no retinopathy or into an intermediate-risk patient cohort with mild retinopathy (each cohort $n=500$ ). Screening intervals were 36 months for the lowrisk cohort and 18 months for the intermediaterisk cohort.

Results: The 1000 subjects enrolled in the study had a median age of 68 (interquartile range 12 ) years and $60.4 \%$ were men. At the follow-up screening visit, data on 102 subjects were not included in the analysis of adherence rate due to death, severe systemic illness, other concurrent eye disease or migration. Among the 898 remaining subjects, adherence to the screening program was $93.7 \%(413 / 443)$ in the 36 -month group and $98.3 \%(449 / 455)$ in the 18 -month group $(p<0.0001)$. Non-adherence decreased with increasing age (odds ratio 0.92, 95\% confidence interval 0.888-0.954, $p=0.0005)$. At follow-up, 65 subjects showed progression of retinopathy; none had worse than moderate retinopathy. Risk factors for DR and treatment for hyperglycemia, hypertension and hyperlipidemia were compared among subjects in the low-risk cohort: non-adherent subjects did not differ from their adherent counterparts without progression of DR, but the former had a shorter duration of diabetes and higher diastolic blood pressure than adherent 
subjects with progression of DR $(4.5$ vs. 7.5 years, $p=0.007$; and 80 vs. $75 \mathrm{mmHg}$, $p=0.02$, respectively).

Conclusion: The results suggest that screening DR at extended intervals can be achieved with high adherence rates without compromising patient safety. However, younger subjects and those at higher risk of progression may require extra attention.

Keywords: Adherence; Attendance; Diabetes mellitus; Diabetic retinopathy; Screening

\section{Key Summary Points}

\section{Why carry out this study?}

The increasing prevalence of diabetes places stress on screening programs for diabetic retinopathy (DR).

A strategy of extended intervals in screening programs for DR would ease the workload, but there has been hesitation in implementation due to concerns of comprising patient adherence and safety.

No previous study has reported on how subjects with diabetes adhere to DR screening at different interval lengths.

\section{What was learned from the study?}

There was high patient adherence to DR screening with extended intervals of up to 36 months.

Non-adherence decreased with increasing age.

Incidence and progression of DR was low among subjects in the screening program.

Stratifying patients to extended intervals based on absence/presence of mild DR appears to be a safe strategy.

\section{DIGITAL FEATURES}

This article is published with digital features, including a summary slide, to facilitate understanding of the article. To view digital features for this article go to https://doi.org/10.6084/ m9.figshare.13122059.

\section{INTRODUCTION}

Screening for diabetic retinopathy (DR) is recommended by the World Health Organization since such evaluations have been shown to prevent blindness through the timely detection of sight-threatening DR (STDR) that can respond to local eye treatment [1]. Screening intervals of up to 1 or 2 years are generally recommended for patients with type 2 diabetes patients (T2DM) with mild or no DR [2,3], but it has been suggested that extending screening intervals beyond the recommended time spans would be cost-effective and help avoid unnecessary controls [4-7]. However, a common concern among healthcare professionals has been that adherence may be poorer when intervals are longer than 1 year [8-12], although to date no study has reported on how subjects adhere to screening at different interval lengths. Taking into account that most diabetes patients have T2DM and that the majority of these individuals do not have retinopathy and are at low risk of progression to STDR [13], extending control intervals for this population is likely to substantially reduce the workload in screening programs.

We hypothesized that adherence to screening for DR in T2DM would be no worse for individuals at low risk of progression to STDR and scheduled for a longer screening interval than for those at intermediate risk and assigned to a shorter screening interval. To test our hypothesis, we accessed data on two representative cohorts with T2DM assigned to screening at a 36- or 18-month interval, respectively, based on the absence or presence of mild DR. 


\section{METHODS}

\section{Research Design and Subjects}

This retrospective cohort study included 1000 patients participating in the screening program in Värmland County, Sweden. This program represents the only DR screening program performed in the county (population 280,000), where 16,000 individuals have been diagnosed with type 1 diabetes (T1DM) or T2DM. In this screening program, a patient with T2DM is assigned a 36- or 18-month screening interval based on the absence or presence of mild DR, in accordance with guidelines from the Swedish National Board of Health and Welfare [14].

The study followed the tenants of the Declaration of Helsinki and was approved by the Regional Ethical Review Board, Uppsala, Sweden (Registry No. 2017-092). Consent of participants to publish was not necessary as no patient-identifying data were presented.

Inclusion criteria were T2DM, age 1879 years and no or mild DR. Two cohorts (each $n=500$ ) were consecutively selected from patients with T2DM participating in the screening program, with a follow-up visit planned during the same period. Subjects in one cohort had no DR and were subsequently assigned a 36-month screening interval; this cohort was designated the low-risk cohort. Subjects in the other cohort had mild DR and were subsequently assigned an 18-month interval; this cohort was designated the intermediate-risk cohort.

The screening program is provided by Region Värmland which is responsible for the county's publicly funded health care. The program is open to all patients with diabetes from the age of 10 years. The screening is provided at multiple (four different access points) separate locations in the region, and the patients are generally allocated to the closest available screening location. Each screening visit includes dilation of pupils and fundus photography, performed by a specially trained nurse using a Topcon TRC-NW8 retinal camera (Topcon Corp., Tokyo, Japan), which captures two redfree $45-50^{\circ}$ images per eye centered on the fovea and the optic disc. The program uses a telemedicine platform, with images sent electronically to one center within the region for evaluation by trained graders using IMAGENet i-base software version 3.18.3 (Topcon Europe Medical BV, Essebaan, The Netherlands). The images are graded according to the International Clinical Diabetic Retinopathy (ICDR) Severity Scale [15]. Each patient's diabetologist/general practitioner receives feedback via the electronic medical records (EMR) system on the current grade of the patient's retinopathy. Any patient failing to attend a screening visit is sent a letter of reminder to schedule a new appointment. The treating diabetologist/general practitioner is also notified via EMR, encouraging a mutual dialogue with the patient on diabetes and DR.

The endpoint for our study was defined as the proportion of patients attending the followup screening appointment. Attendance rate was calculated as the total proportion of subjects present at the follow-up screening visit, regardless of reasons for loss to follow-up. Adherence rate was calculated after subtracting patients not attending due to the following specified reasons: death, severe systemic illness, rescheduling due to other concurrent eye disease or migration from the region. Patients without these specified reasons for not attending were defined as non-adherent if they had failed to reschedule within 3 months after the first given appointment. The primary aim was to explore adherence rates in the two cohorts. Secondary aims were to analyze whether the number of previous visits in the program was correlated to non-adherence and to analyze differences in risk factors for DR and treatment for diabetes and complications among non-adherent and adherent subjects.

\section{Data Sources}

Data on the level of retinopathy, the number of previous screening visits and the shortest travel distance from patients' residences to the allocated screening service were acquired from EMR at the grading center. Characteristics such as age, gender, diabetes duration, hemoglobin A1c 
(HbA1c), systolic and diastolic blood pressure (BP) and treatment for diabetes and hypertension were acquired from the Swedish National Diabetes Registry (NDR).

\section{Statistical Analysis}

The sample size was calculated to determine whether adherence at the follow-up visit was not poorer in the low-risk cohort than in the intermediate-risk cohort with an expected adherence of $90 \%$, a power of $80 \%$, a least detectable difference in adherence of $5 \%$ and an attrition rate of $10 \%[16,17]$. Differences in baseline data between the main cohorts were analyzed using $t$ tests or the Mann-Whitney $U$ test. Difference in adherence between the low-risk and intermediate-risk cohorts was tested using the $\mathrm{Chi}^{2}$-test. A multiple logistic regression was performed to analyze if non-adherence was affected by the number of previous screening visits, controlling for demographic factors, including age, gender and distance to screening center. Differences in risk factors for DR and treatment for diabetes, hypertension and hyperlipidemia between non-adherent subjects, adherent subjects without and adherent subject with progression of DR were analyzed using Fisher's exact test or Kruskal-Wallis test with Holm-Bonferroni correction of $p$ values. Where data is not normally distributed, values given are the median and interquartile range (IQR). $p$ values of $\leq 0.05$ were considered to be significant. Analyses were conducted using IBM SPSS Statistics version 26.0 (IBM Corp. Armonk, NY, USA).

\section{RESULTS}

The 1000 T2DM patients included in this study had a median age of 68 (IQR 12) years, and $60.4 \%$ were men. An HbA1c value $<7.0 \%$ $(53 \mathrm{mmol} / \mathrm{mol})$ was present in $57 \%$ of subjects. In total, 381 subjects were $\geq 70$ years, among whom $52.2 \%$ had a blood pressure (BP) $<140$ / $90 \mathrm{mmHg}$. Of the remaining 619 subjects, $41.5 \%$ had a $\mathrm{BP} \leq 130 / 80 \mathrm{mmHg}$. Compared to the patients in the intermediate-risk cohort, those in the low-risk cohort were younger $(p<0.0001$, Mann-Whitney $U$ test), had shorter diabetes duration $(p<0.0001)$, lower HbA1c $(p<0.0001)$ and less pharmacological treatment for diabetes $\left(p<0.0001, \mathrm{Chi}^{2}\right.$-test $)$, hypertension $(p=0.0001)$ or hyperlipidemia $(p<0.0001)$. Baseline characteristics of the patients are presented in Table 1.

Of the 1000 patients enrolled in the study, 864 attended their follow-up screening visit, representing an overall attendance rate of $86 \%$. Of the 136 who did not attend their follow-up screening visit, 102 had specific reasons for nonattendance. Despite the difference in time to follow-up between the low-risk and the intermediate-risk cohort, namely 36 versus 18 months, there was no significant difference between the cohorts in terms of the number of subjects with specified reasons for not attending follow-up (57/500 and 45/500, respectively; $p=0.21$ ) (Fig. 1). After adjusting for the specified reasons for non-attendance, 898 patients were eligible for further analysis of adherence; of these 34 were non-adherent and 864 were adherent, representing an overall adherence rate of $96.2 \%(864 / 898)$. In the low-risk cohort $(n=443)$, the proportion of adherent subjects was $415 / 443$, corresponding to an adherence rate of $93.7 \% \quad(95 \%$ confidence interval [CI] 0.91-0.958). In the intermediate-risk cohort $(n=455)$ the proportion of adherent subjects was $449 / 455$, corresponding to an adherence rate of $98.7 \%$ (95\% CI 0.972-0.995) (Fig. 2a). Based on these findings, a smaller proportion of subjects were adherent in the lowrisk cohort compared to the intermediate-risk cohort ( $p<0.0001, \mathrm{Chi}^{2}$-test).

Too few subjects $(6 / 455)$ were non-adherent in the intermediate-risk cohort to perform any further statistical analyses.

The number of previous screening visits or the travel distance to the allocated screening center did not correlate to non-adherence (Table 2). Age was the only factor significantly associated with non-adherence. Non-adherence decreased with older age (odds ratio [OR] 0.92, 95\% CI 0.888-0.954; $p=0.0005$ ). The median age of non-adherent and adherent subjects was 52 (IQR 18) years and $65(\mathrm{IQR}=13)$ years, respectively (Fig. 2b). 
Table 1 Baseline characteristics of the patients in the low- and intermediate-risk cohorts

\begin{tabular}{lllc}
\hline Patient characteristics & $\begin{array}{l}\text { Low-risk cohort } \\
(\boldsymbol{n}=\mathbf{5 0 0})\end{array}$ & $\begin{array}{l}\text { Intermediate-risk cohort } \\
(\boldsymbol{n}=\mathbf{5 0 0})\end{array}$ & $\boldsymbol{p}$ \\
\hline Age (years) & $65(14)$ & $69(9)$ & $<0.0001$ \\
Male sex (n) & $294(59 \%)$ & $310(62 \%)$ & 0.30 \\
Diabetes duration (years) & $6(8)$ & $13(9)$ & $<0.0001$ \\
HbAlc NGSP (\%) & $6.6(3.5)$ & $7.0(3.7)$ & $<0.0001$ \\
HbAlc IFCC (mmol/mol) & $49(15)$ & $53(17)$ & $<0.0001$ \\
Systolic BP (mmHg) & $134(17)$ & $135(20)$ & 0.65 \\
Diastolic BP (mmHg) & $80(10)$ & $75(10)$ & 0.001 \\
Glucose-lowering treatment (\%) & $374(74.8)$ & $453(90.6)$ & $<0.0001$ \\
Anti-hypertensive treatment (\%) & $357(71.4)$ & $408(81.6)$ & 0.0001 \\
Anti-hyperlipidemia treatment (\%) & $266(53.2)$ & $343(68.6)$ & $<0.0001$ \\
Treatment for hypertension and & $226(45.2)$ & $319(63.8)$ & $<0.0001$ \\
hyperlipidemia $(\%)$ & & $6(5)$ & $<0.0001$ \\
Prior screening visits $(n)$ & $2(2)$ & $22.6(45.2)$ & 0.51 \\
Distance to screening center $(\mathrm{km})$ & $23.5(50.5)$ &
\end{tabular}

Data are presented as the median with the interquartile range (IQR)) unless otherwise specified.

$B P$ Blood pressure, HbAlc hemoglobin Alc (glycosylated hemoglobin), IFCC International Federation of Clinical Chemistry and Laboratory Medicine, NGSP National Glycohemoglobin Standardization Program

Both cohorts had their follow-up screening visit during the same 6-month period. On average, subjects in the low-risk cohort were screened $12 \pm 42$ days after the planned 3-year follow-up visit and subjects in the intermediaterisk group were screened $12 \pm 39$ days after the intended 18-month follow-up visit. One year after the scheduled follow-up, only two of the 34 non-adherent subjects had rescheduled a retinal examination.

Photographs taken at the follow-up visit were ungradable due to media opacities for only one subject in the intermediate-risk group. Incidence/progression of DR had occurred in 65 patients. In the low-risk cohort, with no DR at baseline, retinopathy occurred in 40/415 subjects $(9.6 \%)$, of whom 36 had mild and four had moderate DR; none of these 40 subjects exhibited any signs of diabetic macular edema. In the intermediate-risk cohort, with mild DR at baseline, progression of retinopathy was observed in 25/449 subjects (5.6\%), with all 25 having progressed to moderate DR. In four of these 25 patients, hard exudates were evident in the macular region. At a subsequent evaluation, those subjects had normal visual acuity (20/20), showed no evidence of retinal thickening and did not require local eye treatment.

Risk factors for DR and treatment for hyperglycemia, hypertension and hyperlipidaemia were compared among non-adherent subjects whose DR status was unknown, adherent subjects without DR and adherent subjects with DR, all in the low-risk cohort (Table 3). Nonadherent subjects differed from adherent subjects with DR in terms of median diabetes duration (4.5 vs. 7.5 years, respectively; $p=0.009$, Kruskal-Wallis test), median diastolic BP (80 vs. $75 \mathrm{mmHg}$, respectively; $p=0.009$ ) and the proportion of subjects with anti-hyperlipidemic treatment (36 vs. 78\%, respectively; $p=0.006$ ). 
Low-risk cohort

Intermediate-risk cohort

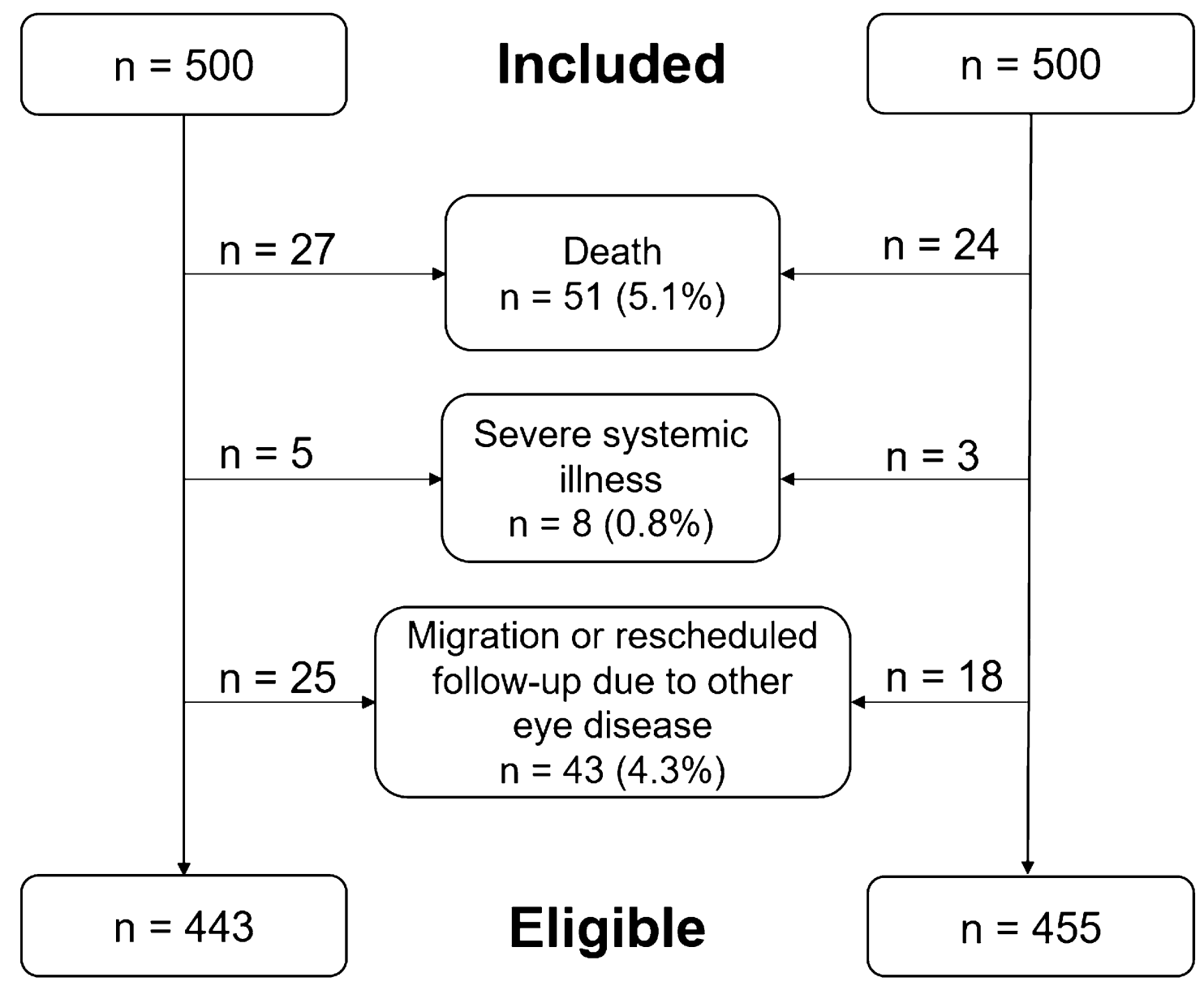

Fig. 1 Number of included, excluded and eligible patients in the low-risk and intermediate-risk cohorts assessed for adherence to follow-up visit in screening programs with a 36- and 18-month follow-up interval, respectively

Non-adherent subjects did not differ from adherent subjects in terms of HbA1c level or systolic BP. Adherent subjects with DR differed from those without DR in terms of duration of diabetes (median 7.5 vs. 5.0 years; $p=0.005$ ) and HbA1c (6.9 [52] vs $6.5 \%$ [48 $\mathrm{mmol} / \mathrm{mol}]$, respectively; $p=0.01)$.

\section{DISCUSSION}

Extended intervals for DR screening have been extensively debated due to a lack of information on whether adherence is negatively affected if screening events are performed less frequently [8-12]. We investigated adherence in 1000 patients with T2DM participating in a DR screening program, applying extended intervals of 36 and 18 months for patients at low- or intermediate-risk of DR progression, respectively. The total adherence was $96.2 \%$, and no patient showed progression to more than moderate non-proliferative DR.

The results of this study refuted our hypothesis by showing that adherence was poorer in the low-risk cohort. Nonetheless, our findings do demonstrate that DR screening with extended intervals can be performed on T2DM patients while safely achieving high adherence. This observation supports a transition to screening programs with a more favorable 


\section{a}

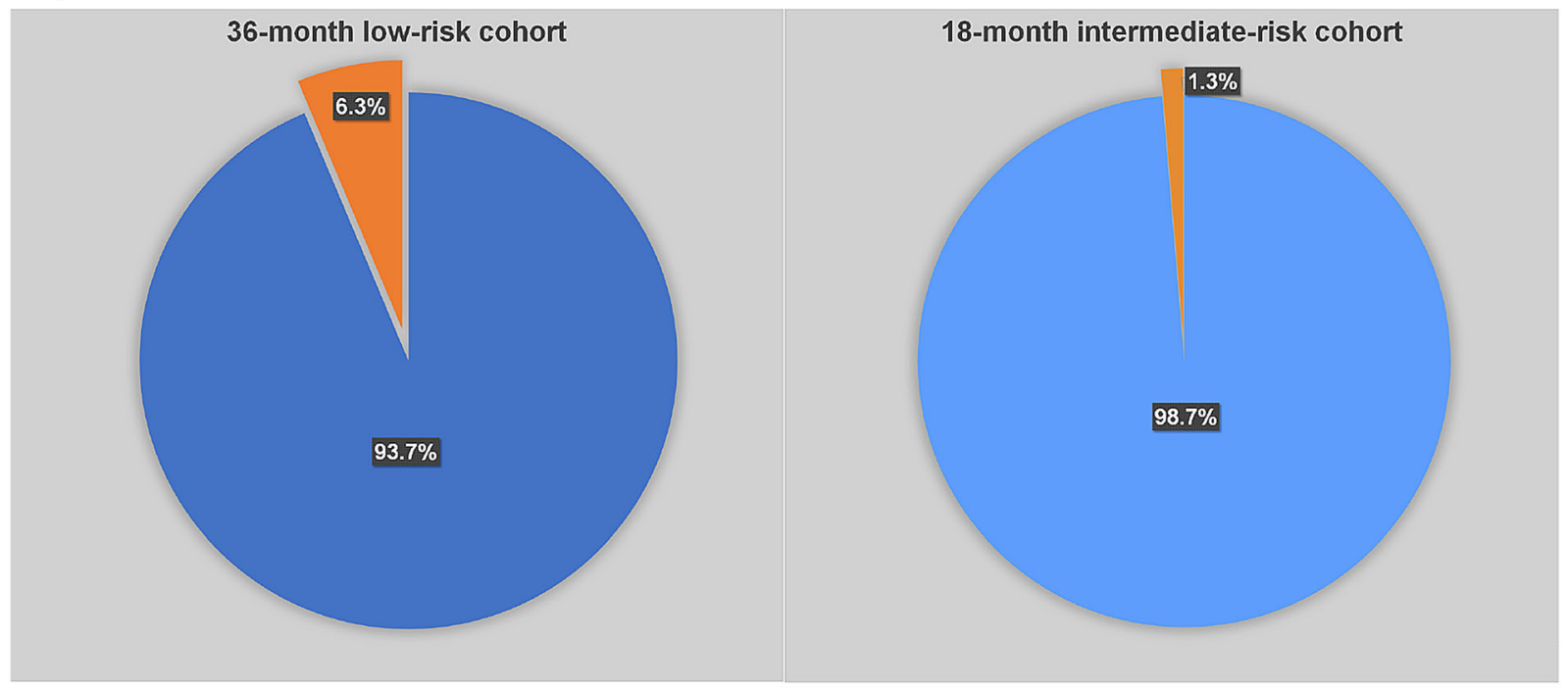

b

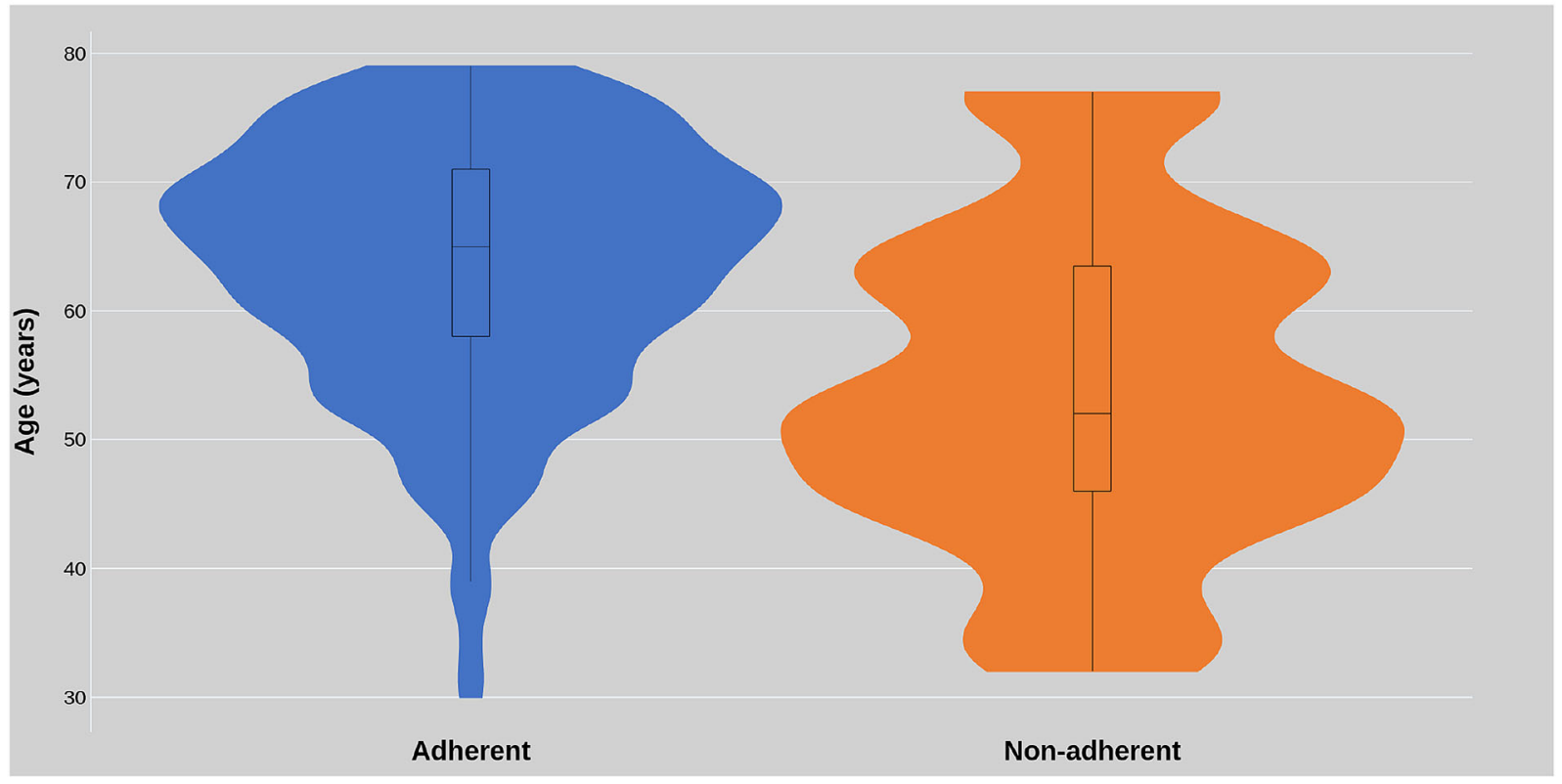

Fig. 2 a The proportion of adherent (blue) and nonadherent (orange) patients participating in the diabetic retinopathy (DR) screening program. The majority of subjects were adherent, but the proportion of nonadherent patients was 5\% higher in the low-risk cohort (without DR at baseline) assigned a 36-month screening interval compared to the intermediate risk cohort (with

cost-utility profile in which unnecessary control visits are avoided.

The adherence rates for the low-risk cohort $(93.7 \%)$ and the intermediate-risk cohort mild DR at baseline) assigned an 18-month screening interval. b Violin plots with overlaid box plots of age in adherent (blue) and non-adherent (orange) patients in the low-risk cohort. Compared to adherent subjects, the nonadherent subjects were younger (median age 52 vs. 65 years, respectively)

(98.7\%) are higher than rates observed in previous investigations, which range from 71 to $91 \%[16,18-21]$. Only two of the cited studies exclusively included patients with T2DM 
Table 2 Factors associated to non-adherence rate in the low-risk cohort

\begin{tabular}{llll}
\hline Factors & Odds ratio & $\mathbf{9 5 \%}$ Confidence interval of non-adherence & $\boldsymbol{p}$ \\
\hline Age (years) & 0.92 & $0.888-0.954$ & 0.0005 \\
Male sex (\%) & 1.175 & $0.52-2.658$ & 0.70 \\
Prior screening visits & 1.042 & $0.807-1.345$ & 0.76 \\
Distance to screening center $(\mathrm{km})$ & 1.007 & $0.998-1.017$ & 0.13 \\
\hline
\end{tabular}

Table 3 Risk factors for diabetic retinopathy and treatment for diabetes, hypertension and hyperlipidemia in non-adherent and adherent subjects in the low-risk cohort

\begin{tabular}{|c|c|c|c|}
\hline \multirow[t]{3}{*}{ Risk factors } & \multicolumn{3}{|l|}{ Low-risk cohort } \\
\hline & \multirow{2}{*}{$\begin{array}{l}\text { Non adherent subjects } \\
\text { DR unknown }(n=28)\end{array}$} & \multicolumn{2}{|l|}{ Adherent subjects } \\
\hline & & No DR $(n=375)$ & DR $(n=40)$ \\
\hline Diabetes duration (years) & $4.5(6.75) \dagger$ & $5(8) \ddagger$ & $7.5(9.25)$ \\
\hline HbAlc NGSP (\%) & $6.7(3.2)$ & $6.5(3.4)$ & $6.9(5.0)$ \\
\hline $\mathrm{HbAlc} \mathrm{IFCC}(\mathrm{mmol} / \mathrm{mol})$ & $50(12)$ & $48(14) \ddagger$ & $52(31)$ \\
\hline Systolic BP (mmHg) & $134(18)$ & $133(17)$ & $135(17)$ \\
\hline Diastolic BP (mmHg) & $80(10) \dagger$ & $80(10)$ & $75(10)$ \\
\hline Glucose-lowering treatment (\%) & $17(61 \%)$ & $285(76 \%)$ & $34(85 \%)$ \\
\hline Anti-hypertensive treatment (\%) & $18(64 \%)$ & $269(72 \%)$ & $33(82 \%)$ \\
\hline Anti-hyperlipidemia treatment (\%) & $10(36 \%) \dagger$ & $201(54 \%)$ & $28(70 \%)$ \\
\hline
\end{tabular}

Data are presented as the median with the IQR in parenthesis or as a number with the percentage in parenthesis $D R$ Diabetic retinopathy

$\dagger$ indicates significant differences at $p<0.05$ between non-adherent subjects and adherent subjects with DR at follow-up. $\ddagger$ indicates significant differences at $p<0.05$ between adherent subjects without DR and adherent subjects with DR

$[16,20]$, but none of the subjects evaluated in those investigations had retinopathy. The authors of one of those two studies reported that $87 \%$ of subjects scheduled for annual examinations had at least one screening event over a 4-year period [20], but they did not provide the specified reasons for non-attendance, which may partly explain the higher rate of adherence in our study. The second study used an extended screening interval of 3 years, and an adherence rate of 91\% was observed [16], which is close to that noted in our investigation. The high adherence rates we report from the screening program in our study may also be explained by having implemented a panel of interventions reported to be favorable for DR screening [22]. These interventions include facilitating retinal examinations by multiple access points, using telemedicine, giving feedback to diabetologists and general practitioners and sending reminders to patients who failed to attend their follow-up visit. Furthermore, the NDR provides open access for benchmarking within the region and across the country [23].

Although adherence at the follow-up visit was excellent in both cohorts, it was 5\% lower 
in the cohort with a 36-month screening interval than in the cohort with an 18-month screening interval (93.7 vs. 98.7\%). In our study, we assessed adherence at the next planned follow-up screening event, and it is possible that the difference in adherence rate will diminish over time, since the intermediate-risk cohort will have to adhere to the screening program twice as often as the low-risk cohort. The difference in adherence may also be explained by age and the absence or presence of retinopathy. Our cohorts were defined based on the absence or presence of mild retinopathy, where those with mild retinopathy were slightly older. Adherence to retinal examinations has been reported to be better in subjects with retinopathy than in those without this diabetic complication [21, 24, 25]. Also, adherence has been found to be higher in patients of older age $[19,24,26]$. In line with these previous findings, we found that non-adherence decreased with older age. We also found no association between adherence and distance to screening center, which is similar to results previously reported, suggesting that the level of accessibility offered in our setting was acceptable $[19,21]$. Adherence was not found to be affected by the number of previous screening visits, suggesting that patients also comply alike to recurrent visits with extended intervals.

The incidence of any retinopathy over the 3 -year screening interval in the low-risk cohort was $9.6 \%$, with none of the affected subjects showing disease progress to STDR. Previous reports on screening DR in patients with T2DM have indicated the incidence of any DR to be between 16.7 and $28 \%$ over 3 years $[5,16]$ and $36 \%$ over 4 years [20]. Glycemic control was only reported in one of these studies [16], and we compared our values with those cited in that investigation: in comparison, fewer patients in the present study were without glucose-lowering drug treatment and a majority of subjects met the treatment targets for glycemic control [27], thus possibly achieving better glycemic control. This may explain the lower incidence and non-occurrence of STDR in our study. Furthermore, BP levels were not reported in any of the earlier studies, and almost half of the patients in our study met the targets for BP, indicating that these patients were well controlled [27].

Our results indicate a very modest progression of retinopathy in both cohorts, suggesting that it can be possible to conduct a safe and effective screening program even with screening at intervals of $>1$ year. With regards to safety in the current healthcare environment, healthcare providers also need to consider risk factors for severe coronavirus disease 2019 (COVID-19) infection as many patients with diabetes have multiple risk factors for COVID-19, such as older age, male gender, obesity and hypertension [28]. In this perspective, extending intervals for screening DR may reduce unnecessary exposure of vulnerable patients to severe acute respiratory syndrome coronavirus 2 (SARS-CoV2).

\section{Strengths and Limitations}

Our study had several strengths. First, the consecutive inclusion of patients provided a representative sample of patients. Second, only subjects with T2DM were included, after considering differences between T1DM and T2DM with regards to demography, incidence of DR and intensity of diabetic treatment and monitoring [29-31]. Third, a clear definition of nonadherence was used, determined as disregarding a screening visit and not making a new appointment within 90 days of the first scheduled follow-up appointment. As only $6 \%$ of the non-adherent subjects had re-entered the screening program 1 year after failing to attend their follow-up visit, the 90-day window for rescheduling reflected non-adherence for at least 1 year. Fourth, assessed clinical parameters were from the NDR. These have high validity [32]. Also, NDR data were complete at a level of $>95 \%$ for any analyzed variable of patient characteristics.

Our findings should also be viewed in light of the limitations to the study. A retrospective design may suffer from selection bias, but such an effect may have been minimized in our study by the consecutive inclusion of patients in each cohort. To avoid any seasonal differences in accessibility to the screening program, the 
follow-up visits for the two cohorts were scheduled during the same period. We consider our catchment area as favorable in terms of minimizing selection bias because this region has only a single provider of DR screening to cover the population and also has a low migration rate [33]. Regional differences in deprivation may also introduce bias, as indicated by previous reports showing poorer adherence to screening among patients with low socioeconomic status [21, 29, 34, 35]. Public health in the studied region is similar or only slightly inferior to the Swedish national average [36]; therefore, we do not believe that the adherence rates in our investigation were overestimated due to socioeconomic and demographic differences. However, our data may not be representative for populations with poorer glycemic control. Also, our study did not include patients with more severe retinopathy or T1DM, or elderly patients aged $\geq 80$ years, and hence it may be valuable to focus on these subgroups in future studies of adherence to DR screening.

\section{CONCLUSIONS}

With an increasing prevalence of diabetes, effective and safe screening for DR is a pressing issue. Considering that $>90 \%$ of all individuals with diabetes have been diagnosed with T2DM, and the majority of these subjects have only mild or no retinopathy [13], extending the screening intervals for these patients will have a substantial positive impact on the effectiveness and costs of screening programs. One strategy to accomplish this is to stratify patients based on retinopathy (i.e. such as the stratification used in the present study). Another potential strategy is to optimize intervals based on individual risk using several risk factors for progression of DR [37, 38]. When such strategies are implemented, prospective studies controlling for age and risk factors for progression of DR could examine whether the length of intervals has any impact on adherence. It is plausible that earlier concerns that extended intervals lead to poorer adherence can turn out to be the opposite, with patients being less adherent if screening is performed too often. However, based on the present study in which real-life data from a well-established screening program were analyzed, it can be concluded that it is possible to screen for DR with extended intervals of up to 3 years and still achieve excellent adherence.

\section{ACKNOWLEDGEMENTS}

The authors thank the Swedish National Diabetes Register (NDR) and the Department of Ophthalmology, Karlstad Hospital, Sweden, for contributing to the acquisition of data.

Funding. The study was funded by grants from the Kamprad Family Foundation for Entrepreneurship, Research \& Charity and the Centre for Clinical Research, Region Värmland, Sweden. The journal's Rapid Service fee was financed by grants from the Kamprad Family Foundation for Entrepreneurship, Research \& Charity.

Authorship. All named authors meet the International Committee of Medical Journal Editors (ICMJE) criteria for authorship for this article, take responsibility for the integrity of the work as a whole, and have given their approval for this version to be published.

Authorship Contributions. AS organized the project, collected the data, analyzed the data, wrote the manuscript draft, edited the manuscript and approved the final draft. JJ edited the manuscript and approved the final draft. K-JH accessed the data from the NDR, edited the manuscript and approved the final draft. JJ and $\mathrm{K}-\mathrm{JH}$ designed the project. $\mathrm{K}-\mathrm{JH}$ is the guarantor of the study.

Prior Presentation. Parts of this work were presented at the 28th Meeting of the European Association for the Study of Diabetes Eye Complication Study Group, Belfast, Northern Ireland, May 25-26th, 2018. 
Disclosures. The authors Ali Sharif, Johan Jendle and Karl-Johan Hellgren declare that they have nothing to disclose.

Compliance with Ethics Guidelines. The study followed the tenants of the Declaration of Helsinki and was approved by the Regional Ethical Review Board, Uppsala, Sweden (Registry No. 2017-092).Consent of participants to publish was not necessary as no patient-identifying data were presented.

Data Availability. The datasets generated during and/or analyzed during the current study are available from the corresponding author on reasonable request.

Open Access. This article is licensed under a Creative Commons Attribution-NonCommercial 4.0 International License, which permits any non-commercial use, sharing, adaptation, distribution and reproduction in any medium or format, as long as you give appropriate credit to the original author(s) and the source, provide a link to the Creative Commons licence, and indicate if changes were made. The images or other third party material in this article are included in the article's Creative Commons licence, unless indicated otherwise in a credit line to the material. If material is not included in the article's Creative Commons licence and your intended use is not permitted by statutory regulation or exceeds the permitted use, you will need to obtain permission directly from the copyright holder. To view a copy of this licence, visit http://creativecommons.org/licenses/bync/4.0/.

\section{REFERENCES}

1. World Health Organization. Prevention of blindness from diabetes mellitus-report of a WHO consultation. 2006. https://www.who.int/diabetes/ publications/prevention_diabetes2006/en/. Accessed 23 Aug 2020.

2. International Council of Ophthalmology. Guidelines for diabetic eye care. 2017. http://www.icoph. org/downloads/ICOGuidelinesforDiabeticEyeCare. pdf. Accessed 23 Aug 2020.
3. Solomon SD, Chew E, Duh EJ, et al. Diabetic retinopathy: a position statement by the American Diabetes Association. Diabetes Care. 2017;40: 412-8.

4. Vijan S, Hofer TP, Hayward RA. How often should patients with diabetes Be screened for retinopathy? JAMA. 2000;284:437-9.

5. Younis N, Broadbent DM, Vora JP, Harding SP. Incidence of sight-threatening retinopathy in patients with type 2 diabetes in the Liverpool Diabetic Eye Study: a cohort study. Lancet. 2003;361: 195-200.

6. Scanlon PH, Aldington SJ, Leal J, et al. Development of a cost-effectiveness model for optimisation of the screening interval in diabetic retinopathy screening. Health Technol Assess. 2015;19:1-116.

7. Lund SH, Aspelund T, Kirby P, et al. Individualised risk assessment for diabetic retinopathy and optimisation of screening intervals: a scientific approach to reducing healthcare costs. Br J Ophthalmol. 2016;100:683-7.

8. Fong DS, Gottlieb J, Ferris FL 3rd, Klein R. Understanding the value of diabetic retinopathy screening. Arch Ophthalmol. 2001;119:758-60.

9. Klein R. Screening interval for retinopathy in type 2 diabetes. Lancet. 2003;361:190-1.

10. Echouffo-Tcheugui JB, Ali MK, Roglic G, Hayward RA, Narayan KM. Screening intervals for diabetic retinopathy and incidence of visual loss: a systematic review. Diabetes Med. 2013;30:1272-92.

11. Taylor-Phillips S, Mistry H, Leslie R, et al. Extending the diabetic retinopathy screening interval beyond 1 year: systematic review. Br J Ophthalmol. 2016;100:105-14.

12. Rosenberg JB, Tsui I. Screening for diabetic retinopathy. N Engl J Med. 2017;376:1587-8.

13. Yau JW, Rogers SL, Kawasaki R, et al. Global prevalence and major risk factors of diabetic retinopathy. Diabetes Care. 2012;35:556-64.

14. Swedish National Board of Health and Welfare. National guidelines for diabetes treatment (Swedish). 2018. https://www.socialstyrelsen.se/ globalassets/sharepoint-dokument/artikelkatalog/ nationella-riktlinjer/2018-10-25.pdf. Accessed 23 Aug 2020.

15. Wilkinson CP, Ferris FL 3rd, Klein RE, et al. Proposed international clinical diabetic retinopathy and diabetic macular edema disease severity scales. Ophthalmology. 2003;110:1677-82. 
16. Agardh E, Tababat-Khani P. Adopting 3-year screening intervals for sight-threatening retinal vascular lesions in type 2 diabetic subjects without retinopathy. Diabetes Care. 2011;34:1318-9.

17. Sealed Envelope Ltd. Power calculator for binary outcome non-inferiority trial. 2012. https://www. sealedenvelope.com/power/binary-noninferior/. Accessed 23 Aug 2020.

18. Scanlon PH, Carter SC, Foy C, Husband RF, Abbas J, Bachmann MO. Diabetic retinopathy and socioeconomic deprivation in Gloucestershire. J Med Screen. 2008;15:118-21.

19. Leese GP, Boyle P, Feng Z, Emslie-Smith A, Ellis JD. Screening uptake in a well-established diabetic retinopathy screening program: the role of geographical access and deprivation. Diabetes Care. 2008;31:2131-5.

20. Thomas RL, Dunstan F, Luzio SD, et al. Incidence of diabetic retinopathy in people with type 2 diabetes mellitus attending the Diabetic Retinopathy Screening Service for Wales: retrospective analysis. BMJ. 2012;344:e874.

21. An J, Niu F, Turpcu A, Rajput Y, Cheetham TC. Adherence to the American Diabetes Association retinal screening guidelines for population with diabetes in the United States. Ophthalmic Epidemiol. 2018;25:257-65.

22. Lawrenson JG, Graham-Rowe E, Lorencatto F, et al. What works to increase attendance for diabetic retinopathy screening? An evidence synthesis and economic analysis. Health Technol Assess. 2018;22: $1-160$.

23. Eliasson B, Gudbjornsdottir S. Diabetes care-improvement through measurement. Diabetes Res Clin Pract. 2014;106(Suppl 2):S291-294.

24. Schoenfeld ER, Greene JM, Wu SY, Leske MC. Patterns of adherence to diabetes vision care guidelines: baseline findings from the Diabetic Retinopathy Awareness Program. Ophthalmology. 2001;108:563-71.

25. Dervan E, Lillis D, Flynn L, Staines A, O'Shea D. Factors that influence the patient uptake of diabetic retinopathy screening. Ir J Med Sci. 2008;177: 303-8.

26. Stratton IM, Aldington SJ, Taylor DJ, Adler AI, Scanlon PH. A simple risk stratification for time to development of sight-threatening diabetic retinopathy. Diabetes Care. 2013;36:580-5.
27. International Diabetes Federation Clinical Guidelines Task Force 2012. Global guideline for type 2 diabetes. 2012. https://www.iapb.org/wp-content/ uploads/Global-Guideline-for-Type-2-Diabetes-IDF2012.pdfAccessed 23 Aug 2020.

28. Albitar O, Ballouze R, Ooi JP, Sheikh Ghadzi SM. Risk factors for mortality among COVID-19 patients. Diabetes Res Clin Pract. 2020;166:108293.

29. Fong DS, Aiello L, Gardner TW, et al. Retinopathy in diabetes. Diabetes Care. 2004;27(Suppl 1):S84-87.

30. Lind M, Svensson AM, Kosiborod M, et al. Glycemic control and excess mortality in type 1 diabetes. N Engl J Med. 2014;371:1972-82.

31. Tancredi M, Rosengren A, Svensson AM, et al. Excess mortality among persons with type 2 diabetes. N Engl J Med. 2015;373:1720-32.

32. Emilsson L, Lindahl B, Köster M, Lambe M, Ludvigsson JF. Review of 103 Swedish Healthcare Quality Registries. J Intern Med. 2015;277:94-136.

33. Statistics Sweden. Migration by region, age and sex. Years 1997-2018. 2019. http://www.statistikdata basen.scb.se/pxweb/en/ssd/START_BE_BE0101_ BE0101J/Flyttningar97. Accessed 23 Aug 2020.

34. Lu Y, Serpas L, Genter P, Anderson B, Campa D, Ipp E. Divergent perceptions of barriers to diabetic retinopathy screening among patients and care providers, Los Angeles, California, 2014-2015. Prev Chronic Dis. 2016;13:E140.

35. Moreton RBR, Stratton IM, Chave SJ, Lipinski H, Scanlon PH. Factors determining uptake of diabetic retinopathy screening in Oxfordshire. Diabet Med. 2017;34:993-9.

36. Public Health Agency of Sweden. Öppna Jämförelser folkhälsa Open comparisons public health 2019 (Swedish). 2019. https://www.folkhalso myndigheten.se/contentassets/5341a2539ca24c2aa 744cbaf3f19a723/faktablad-oppna-jamforelser-201918076-1.pdf/. Accessed 23 Aug 2020.

37. Aspelund T, Thornorisdottir O, Olafsdottir E, et al. Individual risk assessment and information technology to optimise screening frequency for diabetic retinopathy. Diabetologia. 2011;54:2525-32.

38. Eleuteri A, Fisher AC, Broadbent DM, et al. Individualised variable-interval risk-based screening for sight-threatening diabetic retinopathy: the Liverpool Risk Calculation Engine. Diabetologia. 2017;60:2174-82. 